\section{Aspects of visual and acoustic imagery $* \dagger$}

\author{
ROBERT J. WEBER and JOE KELLEY \\ Oklahoma State University, Stillwater, Okla. 74074
}

Rates of processing imagined letters for visual properties (VP) and for acoustic properties (AP) are assessed. The most rapid imaginal conditions show that letters of an alphabetic list are processed at almost 2 per second for both visual and acoustic properties. Indirect evidence is presented to show that implicit verbal control of letter sequencing may be involved for both visual and acoustic imagination when processing alphabetic lists, thus revealing a link between visual and verbal coding.

The purposes of this study are to compare processing rates for visual and acoustic imagination, to compare rates of abstracting visual and acoustic properties from imagined vs perceptual representations of letters, and to show by manipulation of response mode and representation the possibility of verbal control of visual image sequencing. Rates of processing letters in visual imagination have been previously investigated (Weber \& Bach, 1969; Weber \& Castleman, 1970), and the sequencing problem has been dealt with at some length by Paivio (1971). The sequencing problem pertains to how, in a serial list of visual images, the $S$ gets from one visually imagined form to another. In the Weber et al studies, Ss claimed, subsequent to the experiment, that it was necessary to say implicitly each letter before visualizing it. It is hypothesized here that the series of visually imagined letters of the alphabet is verbally ordered in the sense of S's saying implicitly each successive letter before visualizing it. If so, then we have a potentially important interrelationship between our two most important symbolic codes, visual imagery and verbal processes (Paivio, 1971). Investigation of these foregoing problems seems to require an objective method of assessing the existence of visual imagery.

Following Weber \& Castleman (1970, Experiment 2), it is possible to employ objective criteria of imagining letters and to assess the rate at which letters are processed in imagination. These investigators had Ss classify imagined lowercase letters on the basis of height. Some letters are vertically small $(a, c, e, i, \ldots, z)$ and other letters are vertically large $(b, d, f, g$, $\ldots, y)$. The Ss were instructed to imagine visually the successive alphabetic letters and to classify each

*Send reprint requests to Robert J. Weber, Dept. of Psychology, Oklahoma State University, Stillwater, Okla. 74074.

TThis study was supported in part by the Research Foundation, Oklahoma State University, Stillwater, Okla. successive letter for its vertical height. The Ss reported visually imagining and were able to correctly classify the letters, using a verbal response ("small," "large") at a rate of about 1 letter per second. The rate differences for the objective and subjective procedures require comment. If Ss did, in fact, say implicitly each successive letter before visualizing it, then we would expect that a verbal classification response would compete for processing capacity with the covert verbal sequencing. If this is the case, then processing rate ought to be faster with a nonverbal classification response. Thus, if Ss are to give either a verbal yes/no or a written line/dot equivalent $(/,$.$) in responding to each$ imagined letter, we would expect slower rates for the verbal response mode than for a written response mode if verbal control of the sequence is required. This would be due to competition for verbal processing space on the part of both the overt verbal yes/no response and the implicit verbal control of sequencing.

The general technique of abstracting from images the correlates of physical stimuli also can be applied to the study of acoustic imagination. Letter names possess acoustic properties that can be used as objective indicators of acoustic imagination. Thus, some letter names have a long e sound, /i/ $(b, c, d, e, \ldots, z)$, and other letter names do not $(a, f, h, \ldots, y)$. If $S$ can correctly classify successive alphabetic letters according to their acoustic properties without actually saying them aloud, we can be reasonably sure that acoustic imagination is at work. When doing this task, there is again the strong impression of saying each letter prior to extracting its acoustic property. This may be necessary only for producing and abstracting the acoustic property of the imagined letter, but it may also have a sequential control function, as previously conjectured for visual imagery.

In some preliminary work, we have found the actual rate of letter classification to be very similar for visual and acoustic properties. But, subjectively, visual and acoustic imagination are very different. Accordingly, we have sought to find at least one objective variable that clearly separates them. The variable selected was imagined vs perceptual representation of the alphabetic sequence. It was reasoned that letters visually present (percepts) would have their visual properties immediately available for abstraction, whereas their acoustic properties would be no more available than in imagination. In addition, when the letters are visually present, as percepts, the sequencing problem could be handled by eye movements rather than by implicit verbalization. Hence, the differences in rate between written and verbal response modes should diminish.

\section{SUBJECTS}

Twenty undergraduate volunteers were paid for their participation. One additional $S$ was discarded for inability to do the task. Ten Ss were assigned at random to each of two between-Ss conditions.

\section{DESIGN AND PROCEDURE}

The design consisted of a factorial arrangement of two methods of representation (image, percept) by two properties (visual, acoustic) by two response modes (spoken, written). Response mode was between Ss, and the other two factors were within Ss. Representation could either be imaginal, meaning that the $\mathrm{Ss}$ somehow implicitly generated the successive alphabetic letters, or perceptual, meaning that a printed lowercase serial list of the alphabet was visually present before the $S$ while he made his responses. The properties of the letters were either visual (in which case $S$ had to distinguish between vertically large and vertically small letters of the lowercase alphabet) or acoustic (in which case $S$ had to distinguish between letters with the long e sound, $/ \mathrm{i} /$, in their names from those that did not). Vertically large letters and long e letters were to be classified as yeses, and the other letters were to be classified as nos. For the spoken response mode, $S$ said yes or no for each successive letter of the alphabet, as he classified it for its properties. Thus, in the visual property-image condition, $\mathrm{S}$ would emit a string of the following sort: no, yes, no, yes, ..., no, because the successive letters of the alphabet $(a, b$, $c, d, \ldots, z)$ have that pattern of yes/no classifications of vertical height. If the letters were to be processed for their acoustic properties, the corresponding string of yes/nos would be: no, yes, yes, yes, .... yes. When all letters are so considered, there are, for visual properties, 12 yes and 14 no letters. ${ }^{1}$ For acoustic 
Table 1

Seconds to Process Alphabet $(N=10$ for Each Condition)

Response Mode

\begin{tabular}{|c|c|c|c|c|c|}
\hline \multirow{2}{*}{\multicolumn{2}{|c|}{$\begin{array}{c}\text { Representation } \\
\text { and Property }\end{array}$}} & \multicolumn{2}{|c|}{ Spoken } & \multicolumn{2}{|c|}{ Written } \\
\hline & & Mean & $\mathrm{SE}_{\mathbf{M}}$ & Mean & $\mathrm{SE}_{\mathrm{M}}$ \\
\hline Image & $\begin{array}{l}\text { AP } \\
\text { VP }\end{array}$ & $\begin{array}{l}22.41 \\
21.42\end{array}$ & $\begin{array}{l}4.16 \\
3.21\end{array}$ & $\begin{array}{l}15.60 \\
14.86\end{array}$ & $\begin{array}{l}5.66 \\
3.51\end{array}$ \\
\hline Perçept. & $\begin{array}{l}\text { AP } \\
\text { VP }\end{array}$ & $\begin{array}{l}15.78 \\
12.53\end{array}$ & $\begin{array}{l}2.29 \\
1.57\end{array}$ & $\begin{array}{l}15.16 \\
12.35\end{array}$ & $\begin{array}{l}4.54 \\
2.90\end{array}$ \\
\hline
\end{tabular}

properties, there are 9 yes and 17 no letters. Hence, there is somewhat less response uncertainty for acoustic than for visual properties. For the written response conditions, the same classification was employed, except that a vertical line and dot were chosen to correspond to yes and no, respectively. The $S$ wrote his response on a blank $81 / 2 \times 11$ in. sheet, but he did not visually monitor his writing.

The nature of the acoustic and visual properties of letters was pointed out to $\mathrm{S}$, as well as the particular response mode he was to use. He was then given four practice trials, one for each condition. He was told not to make any errors. Later, during the experiment proper, if an error was made, it was pointed out to $S$ at the end of the trial. This procedure leads to low error rates (Weber \& Castleman, 1970), and errors are not further considered in this study.

The order of presentation of the image-percept by visual-acoustic property conditions was random within each of six blocks. There were four different random lists of the six blocks. The beginning of a trial began with the E's designation of the trial as percept/image and the presentation of a card indicating whether visual or acoustic properties were to be processed. Response time for processing the 26 letters of the alphabet was the dependent variable. The response time interval began with presentation of the card and ended with S saying, "Stop," after completing the last letter of the alphabet. Times were recorded on a Standard Electric clock to the nearest $.01 \mathrm{sec}$. The $\mathrm{S}$ was not given temporal feedback, but he was urged to go as rapidly as possible. There was about a 30-sec interval between successive trials while the $E$ recorded the response time and reset the clock.

\section{RESULTS AND DISCUSSION}

Table 1 shows mean times to process the 26 letters of the alphabet as a function of conditions. Each mean is based on 10 Ss and six trials. The $\mathrm{SE}_{\mathrm{M}}$ is a between-Ss measure of variation. Significance tests for main effects indicate the following: Response time is greater for image than for percept representation, $\mathrm{F}(1,18)=121.84, \mathrm{p}<.01$. Response time is greater in the spoken than in the written mode, $F(1,18)=5.53$, $\mathrm{p}<.05$. And response time is greater in the acoustic than in the visual property conditions, $F(1,18)=15.85$, $\mathrm{p}<.01$.

The findings for main effects must be qualified, however. Perhaps of most interest are the significant two-way interactions: Response Mode by Image-Percept Representation, $F(1,18)$ $=56.57, \mathrm{p}<.01 ;$ and Visual-Acoustic Property by Representation, $\mathrm{F}(1,18)=$ $30.77, p<.01$. The significant Response Mode by Representation interaction indicates that the differences between spoken and written responses were much greater in the image than in the percept conditions. This is to be expected if verbal sequential control is involved in the image conditions. In that case, the verbal control process would compete for processing space with the spoken yes/no response. The significant Property by Representation interaction indicates that the difference between visual and acoustic property processing time is greater for percept than for image representation. Again, this is to be expected, since visual properties are directly available in the percept representation, whereas acoustic properties are not directly available. The Property by Representation interaction illustrates clearly that visual and acoustic imagination are separate processes in accord with our subjective impressions. Neither the Response Mode by Property nor the triple interaction approached significance at the .05 level.

With regard to sequential control, the objective results are consistent with the subjective impression that $S$ implicitly says each successive alphabetic letter before visualizing it and abstracting its spatial height property or before "hearing" it and abstracting its acoustic sound property. For the image conditions, when a spoken yes/no response is required, it competes for verbal processing capacity with the implicit speech generation of the alphabetic sequence. The written response actually leads to more rapid processing because it does not compete for processing capacity with the implicit verbal generation of the sequence. In a long serial list there is, then, evidence for an important interrelation of visual and implicit verbal codes. But this relation does not hold for the percept conditions, where sequencing between successive letters is no longer under verbal control. The sequencing problem is solved simply by moving the eyes from one successive letter form to another. Thus, the difference between spoken and written response time disappears in the percept conditions. Of course, this argument applies only to a long ordered string like the alphabet. Undoubtedly, visual imagination for some kinds of materials is lacking in a verbal sequencing component.

Several final comments are in order. First, when the means of Table 1 are converted to a rate measure, the most rapid image condition at 1.75 letters per second is for visual properties with a written response. This is very comparable to previous subjective measures of visual imagery (Weber \& Bach, 1969; Weber \& Castleman, 1970 , Experiment 1), which neither required overt letter-by-letter responding nor had the additional within-S acoustic property task. On the basis of this comparison, we can be reasonably sure that the written response does not appreciably interfere with and slow down visual image representation. Second, the most rapid imagined acoustic property condition also occurred with the written response mode and was 1.67 letters per second. This is in marked contrast to the approximately 6/sec rate of saying the letters implicitly (Weber \& Castleman, 1970). Thus, there is something quite different in "pure" visualization (without letter-by-letter overt responding) as compared to the "pure" verbalization (saying the successive letters silently to one's self). But this difference tends to disappear when each individual letter must be overtly responded to, suggesting a complex set of relationships. Third, the unequal distribution of yes/no letters for the visual and acoustic property tasks. probably gives rise to a conservative difference. Thus, if yes/no uncertainty were equal for the two tasks, the acoustic property task might have been even slower.

\section{REFERENCES}

PAIVIO, A. Imagery and verbal processes. New York: Holt, Rinehart \& Winston. 1971 .

WEBER, R., \& BACH, M. Visual and speech imagery. British Journal of Psychology, $1969,60,199-202$.

WEBER, R. \& CASTLEMAN, J. The time it takes to imagine. Perception \& Psychophysics, $1970,8,165-168$

$$
\text { NOTE }
$$

1. A few letters, such as "i," are ambiguous, but once specified by $E$. there is no further difficulty. 